\title{
Atrial waveform analysis using esophageal long-term electrocardiography reveals atrial ectopic activity
}

\author{
Andreas Haeberlin • Thomas Niederhauser • \\ Hildegard Tanner $\cdot$ Rolf Vogel
}

Received: 20 March 2012/Accepted: 10 May 2012/Published online: 22 May 2012

(C) Springer-Verlag 2012

\section{Sirs:}

A 59-year-old male patient without the history of heart disease was referred for coronary angiography due to typical chest pain and signs of myocardial ischemia during exercise stress test.

On admission, the patient was in sinus rhythm. After successful PCI, the patient was enrolled in a study to investigate esophageal long-term electrocardiography (eECG) comprising simultaneous rhythm monitoring with surface and esophageal leads during $16 \mathrm{~h}$. The surface ECG (sECG) was recorded using a two-channel Holter ECG recorder with a sampling frequency of $1,024 \mathrm{~Hz}$ allowing continuous rhythm monitoring for 7 days (Lifecard CF, Spacelabs Healthcare, USA). The eECG was recorded using an esophageal ECG electrode (Esosoft 6S, FIAB, Italy) connected to a dedicated two-channel eECG recorder - a proprietary development-with a sampling frequency of $512 \mathrm{~Hz}$. We registered two bipolar channels (interelectrode spacings of 60 and $15 \mathrm{~mm}$ ). The eECG was well tolerated; the patient reported only a slight pharyngeal foreign body feeling without any impact on his common daily activities such as eating, talking and sleeping.

ClinicalTrials.gov Identifier: NCT01436344.

A. Haeberlin $\cdot$ H. Tanner

Department of Cardiology, Bern University Hospital, Bern, Switzerland

A. Haeberlin · T. Niederhauser · R. Vogel ARTORG Cardiovascular Engineering,

University of Bern, Bern, Switzerland

R. Vogel $(\bowtie)$

Department of Cardiology, Bürgerspital Solothurn,

4500 Solothurn, Switzerland

e-mail: rolf.vogel@spital.so.ch
The sECG was analyzed using an automatic beat classifier system (Pathfinder, Spacelabs Healthcare, USA). The software correctly detected five atrial premature beats (APB's) but no other atrial arrhythmias. In the eECG, we unmasked another 40 APB's, as well as 178 episodes with atrial ectopic rhythm [mean duration \pm SD $25 \pm 31$ beats (Fig. 1)].

This report illustrates the limited sensitivity of surface long-term ECG for atrial rhythm disorders. For the sECG, software-based automatic classification of atrial arrhythmias is accomplished by calculating the ratio of the R-Rinterval to be classified and the preceding $\mathrm{R}-\mathrm{R}$-interval(s). This quotient is then compared to a coupling interval threshold, e.g., the Pathfinder software uses by default $66 \%$ of the preceding R-R-interval duration. The number of detected atrial arrhythmias strongly depends on this threshold: atrial ectopic beats are only identifiable if they are premature enough as compared to this threshold. The sECG uses R-R-intervals as surrogate markers of the true atrial activity, while morphologic features of atrial signals are not considered. The lack of a direct classifier for atrial arrhythmias is a major limitation of sECG monitoring in cases without evident change in $\mathrm{R}-\mathrm{R}$ cycle duration. Moreover, reliable detection of atrial arrhythmias is of increasing importance for risk stratification, because it has been shown that excessive supraventricular ectopic activity [1] as well as atrial premature beats [2-4] are associated with an increased risk of stroke and atrial fibrillation. Esophageal electrocardiography may provide an elegant way out by offering excellent atrial signals that additionally allow waveform analysis for the direct and reliable classification of atrial rhythm.

Lead movements due to eating or respiration can confound esophageal electrocardiography. We exclude that the morphologic changes are caused by such artifacts for the 

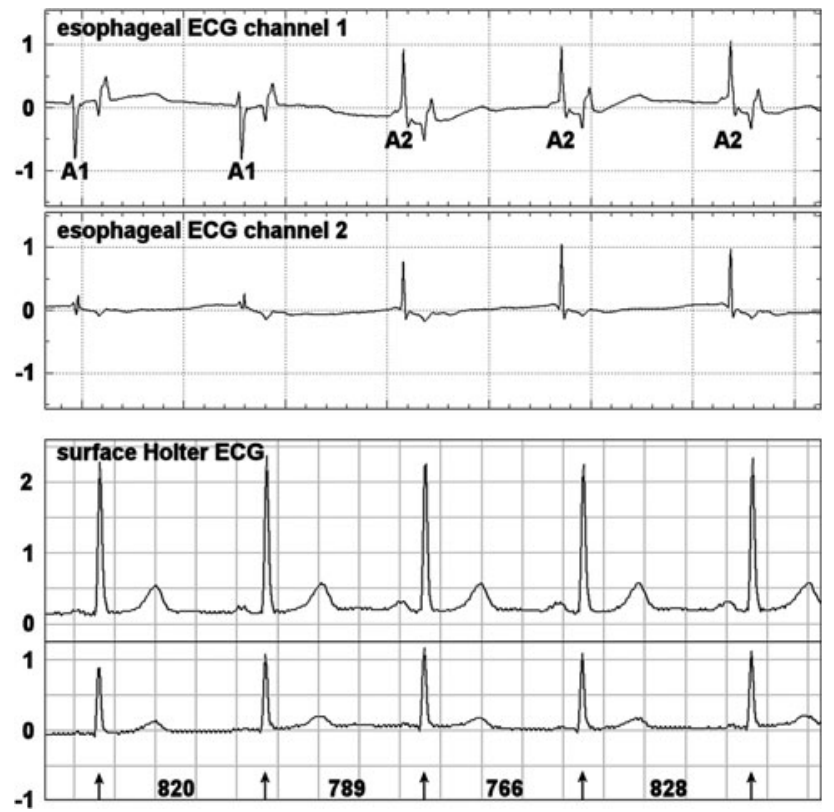

Fig. 1 The two eECG channels (top) show two clearly distinguishable atrial waveforms ( $A 1$ and $A 2)$, whereas their different morphology can be easily overlooked in the simultaneous surface ECG (bottom). Note that the long coupling interval of the first A2 signal (96\% of the cycle duration of the preceding R-R-interval) precludes to detect this ectopic atrial activity based on R-R-cycle information. Channels 1 and 2 show bipolar signals with 60 and $15 \mathrm{~mm}$ interelectrode spacing, respectively, amplitudes are given in $\mathrm{mV}$, cycle lengths in ms

following reasons: such motion artifacts produce low-frequent baseline shift affecting several cardiac cycles and can be identified easily. Mechanical artifacts would not only affect atrial signals but also ventricular complexes. Furthermore as stochastic events, they would have produced different morphologic patterns, which was not the case. In addition, the two lead registration provided redundancy; particularly the large interelectrode spacing of channel one is insusceptible to mechanical artifacts.

This case report illustrates the difficulties of automated atrial rhythm classification by the SECG and demonstrates that the esophageal approach may improve the yield of long-term recordings with respect to atrial arrhythmias. However, this preliminary finding has to be clarified in a larger study population.

Acknowledgments This work was funded by the Swiss Heart Foundation, the Department of Cardiology, Bern University Hospital and the Commission for Technology and Innovation CTI, Switzerland.

Conflict of interest The authors declare that they have no conflict of interest.

\section{References}

1. Binici Z, Intzilakis T, Nielsen OW, Kober L, Sajadieh A (2010) Excessive supraventricular ectopic activity and increased risk of atrial fibrillation and stroke. Circulation 121(17):1904-1911. doi: 10.1161/CIRCULATIONAHA.109.87498

2. Wallmann D, Tüller D, Wustmann K, Meier P, Isenegger J, Arnold M, Mattle HP, Delacrétaz E (2007) Frequent atrial premature beats predict paroxysmal atrial fibrillation in stroke patients: an opportunity for a new diagnostic strategy. Stroke 38

3. Folkeringa RJ, Hartgers J, Tieleman RG, Gorgels AP, Dassen WR, Crijns HJ (2006) Atrial extrasystoles after exercise predict atrial fibrillation in patients with left ventricular hypertrophy. Heart 92(4):545-546. doi:10.1136/hrt.2005.069542

4. Wallmann D, Tuller D, Kucher N, Fuhrer J, Arnold M, Delacretaz E (2003) Frequent atrial premature contractions as a surrogate marker for paroxysmal atrial fibrillation in patients with acute ischaemic stroke. Heart 89(10):1247-1248 